\title{
Editorials
}

\section{General practice:}

\author{
the heart of the NHS
}

General practice is often hailed as the jewel in the crown of the NHS. ${ }^{1}$ However, a decade of underfunding, increasing patient complexity, issues with recruitment, and rising bureaucracy have led to a loss of its lustre. Amid a drive to move care into the community we continue to have the most stressed GPs in the Western world. ${ }^{2}$ This is doubtless impacting on junior doctors and medical students contemplating a career in general practice, as highlighted by Professor Wass's recent report By Choice - Not by Chance. ${ }^{3}$

It is general practice that makes the NHS one of the world's most cost-effective health services, yet spend on general practice continues to be outstripped by that on hospital outpatient clinics alone. Never before has the need for an optimised version of general practice felt more critical to the long-term sustainability of the NHS. As Roland and Everington write, "If general practice fails, the whole NHS fails. '"

\section{THE GP FORWARD VIEW}

The General Practice Forward View (GPFV) was launched in April 2016 by NHS England and Health Education England, with the support of the RCGP. It is a complex plan for a complex set of issues that have besieged primary care for too long. ${ }^{5} \mathrm{NHS}$ England is backing this plan with a $14 \%$ real-terms increase in funding to support general practice services over 5 years. This is almost double the investment going into other parts of the NHS, and a notable change from a system that has historically prioritised the acute sector. At a time of austerity, this remarkable shift seeks to both stabilise and redesign general practice, and break the annual cycle of rescuing hospital overspends.

Key ingredients of the reinvention include increased uptake of self-care, an expanded and upskilled workforce, investment in estates and technology, and enhanced collaboration between practices and with the wider system. All the while, we must maintain a relentless focus on protecting the essence of general practice: the deepseated connection and continuity at the heart of the doctor-patient relationship.

Turning the tide so that palpable changes are felt in the consulting room will take time - the boiling frog will not notice the pot cool overnight. But steady progress is being made. The challenge lies in ensuring

"Turning the tide so that palpable changes are felt in the consulting room will take time - the boiling frog will not notice the pot cool overnight. But steady progress is being made.

that we keep hope alive long enough for the changes to take effect.

\section{GROWING AND SUPPORTING THE WORKFORCE}

The greatest threat to delivering the GPFV is the instability of practices, often rooted in issues with recruitment and retention. NHS England and Health Education England are working to double the rate of growth in the primary care workforce.

Efforts in the first 10 months have included: increased GP training places, improvements to both the Induction and Refresher and the Retainer schemes, international recruitment pilots, postCCT fellowships, salary supplements in hard-to-recruit areas, increasing numbers of clinical pharmacists, and training for new roles such as GP medical assistants, care navigators, and physician associates. We have also launched a new GP Career Plus scheme, designed to offer flexibility and support for GPs considering leaving general practice. Looking further upstream, medical school places are being increased in the future.

Supplementary measures include support for increasing indemnity costs that have distorted individuals' decisions to work streamlining processes of the Performers List, and a new GP Health Service for GPs and trainees facing burnout. ${ }^{6}$

Although these changes are beginning to have some impact, such as securing a record number of new GP trainees in 2016 , we know there is much more we need to do.

\section{RESCUING PRACTICES WITH THEIR BACKS AGAINST THE WALL}

Although these investments will take time to translate into benefits on the ground, our priority is to rescue practices with their backs against the wall. Overall, nearly 2000 practices will benefit from the support offered through the Vulnerable Practices Fund (E10M) and Practice Resilience Programme funds (E16M in 2016/2017 and $\mathrm{E} 40 \mathrm{M}$ total). Furthermore, the $\mathrm{E} 30 \mathrm{M}$ General Practice Development Programme will start to support a third of all practices this financial year, and includes 400 free leadership-training places a year for those working in primary care. This organisational development programme is being delivered on an unprecedented scale, even by international standards.?

Ultimately, if we are to change the perception of general practice for those on the outside looking in, we need to create a more manageable working day for GPs. At the heart of the conundrum lies reducing the burden of low-value activities that cumulatively challenge delivery of care. This will be fundamental in attracting the increase in workforce capacity we need, and in managing the inexorable rise in patient demand.

We are taking action to reduce external pressures, such as disproportionate regulation, reducing avoidable hospital hand-offs Itransfers of care between
"NHS England and Health Education England are working to double the rate of growth in the primary care workforce ... these changes are beginning to have some impact, such as securing a record number of new GP trainees in 2016. 


\section{"We are undoubtedly at the start of a long and challenging journey. But the destination we are aiming for is one where we rediscover the joy in the consultation, recapture the essence of why we entered the profession, and reaffirm our status as the jewel in the crown of the NHS.}

clinicians), and ensuring we develop meaningful self-care offers for patients. We also need to redefine our role in orchestrating a strengthened primary care team that includes care navigators, medical administrators, clinical pharmacists, physician associates, mental health therapists, community services colleagues, and the voluntary sector. This must be underpinned by technology that offers a step-change in utility to patients, connecting clinicians, and streamlining back-office processes.

Lastly, we must consider working across groups of practices to build resilience, economies of scale, and expanded service delivery offers, as well as improving our ability to integrate with other providers.

\section{WORKING DIFFERENTLY}

Broadly, the GPFV has four key objectives: enhance the patient experience, improve health outcomes, deploy resources more wisely, and create more fulfilling roles for those working in primary care. The role that workforce morale plays in this cannot be overstated, and leaving this unaddressed will jeopardise the whole. Maintaining the value GPs place on their autonomy, sense of purpose, and the opportunity to innovate is key in our design principles.

We must also accommodate the changing ambitions of the different workforce layers: partners wishing to remain autonomous independent contractors, salaried GPs looking for a more stable workload and job security, and the increasing number of GPs seeking greater flexibility by carving out a portfolio career. Optimising the offer to each of these groups will be essential, as well as ensuring there is flexibility to move between these roles at different stages.

The decision by practices in many parts of the country to collaborate in federations and networks shows that the case for working at scale is increasingly understood. ${ }^{8}$ The Primary Care Home movement, alongside the formation of two radically changed models of integration in the form of the Multi-Specialty Community Providers (MCPs) and Primary and Acute Care Systems (PACS), provide further opportunities to reorganise or dissolve organisational boundaries. ${ }^{9}$ Amid these new models exciting opportunities for career progression should emerge, for example, by influencing local service reform and fostering closer relationships with secondary care colleagues.

\section{OUR SUCCESS DEPENDS ON PULLING TOGETHER AS A PROFESSION}

Fourteen years have passed since David Haslam's likening of general practice to the 'heat sink' of the computer: absorbing both risk and workload, and crashing the system if it's taken away. ${ }^{10}$ This will remain a timeless truism.

Beneath the demoralising headlines, there has been a detectable change in the narrative among some GPs. It has moved from disbelief in the commitment described in the GPFV, to a sense of anticipation and impatience over its delivery.

We are undoubtedly at the start of a long and challenging journey. But the destination we are aiming for is one where we rediscover the joy in the consultation, recapture the essence of why we entered the profession, and reaffirm our status as the jewel in the crown of the NHS.

Only hindsight will tell whether we have created a point of inflexion for primary care. But even with the best strategy in hand, our success depends on pulling together as a profession to create a sustainable future for the generation coming through the ranks, and for our patients.

As Simon Stevens's opening of the GPFV stated:

There is arguably no more important job in modern Britain than that of the family doctor. ${ }^{5}$

We must preserve the truth in that.

\section{ADDRESS FOR CORRESPONDENCE}

\section{Arvind Madan}

NHS England, Skipton House, 80 London Road, London SE1 6LH, UK.

E-mail: arvind.madananhs.net

Arvind Madan,

Director of Primary Care and Deputy Medical Director, NHS England, London.

\section{Nishma Manek}

GP Trainee and National Medical Director's Clinical Fellow at NHS England, London.

\section{Simon Gregory,}

Director and Dean of Education and Quality, Midlands and East Health Education England; and GP, King Edward Road Surgery, Northampton.

\section{Provenance}

Commissioned; not externally peer reviewed.

DOI: https://doi.org/10.3399/bjgp17X689965

\section{REFERENCES}

Marshall M. A precious jewel - the role of general practice in the English NHS. N Engl J Med 2015; 372(10): 893-897.

2. Martin S, Davies E, Gershlick B. Under pressure: what the Commonwealth Fund's 2015 international survey of general practitioners means for the UK. London: Health Foundation, 2016. http://wnw. health.org.uk/sites/health/files/UnderPressure.pdf laccessed 6 Mar 2017).

3. Wass V. By choice - not by chance supporting medical students towards future careers in general practice. London: Health Education England and the Medical Schools Council, 2016. https://www.hee.nhs.uk/sites/ default/files/documents/By\%20choice $\% 20$ not $\% 20$ by $\% 20$ chance $\% 20$ web $\% 20$ FINAL.pdf (accessed 6 Mar 2017).

4. Roland M, Everington S. Tackling the crisis in general practice. BMJ 2016; 352: i942.

5. NHS England. General practice forward view. https://www.england.nhs.uk/gp/gpfv/ laccessed 7 Mar 2017).

6. NHS England. Workforce. 2016. https://www. england.nhs.uk/gp/gpfv/workforce/ laccessed 6 Mar 2017).

7. NHS England. Building general practice resilience. 2016 .https://uww.england.nhs.uk/gp/gpfv/ workload/resilience/ laccessed 6 Mar 2017).

8. Ham C. A new deal for general practice: doing things differently, not just more of the same. London: King's Fund, 2015. https://www.kingsfund. org.uk/blog/2015/06/new-deal-general-practicedoing-things-differently-not-just-more-same laccessed 6 Mar 2017).

9. NHS England. Five year forward view. 2014 https://www.england.nhs.uk/wp-content/ uploads/2014/10/5yfv-web.pdf laccessed 6 Mar 2017).

10. Haslam D. Editorials. 'Schools and hospitals' for 'education and health'. BMJ 2003; 326(7383): 234-235. 